\title{
Short Time Asymptotic Behaviour and Large Deviation for Brownian Motion on Some Affine Nested Fractals
}

\author{
Dedicated to Professor Shinzo Watanabe on his 60th birthday
}

By

Takashi KumagaI*

\begin{abstract}
We study so called Varadhan type short time asymptotic estimates of heat kernels and Shilder type large deviation for Brownian motion on some affine nested fractals introduced in [7]. As a corollary to our approach, we obtain sharper estimates of heat kernels for a class of one dimensional diffusion processes studied in [8].
\end{abstract}

\section{§1. Introduction}

In $[21], \mathrm{S}$. Watanabe surveys on short time asymptotic behaviour and on large deviations for one dimensional diffusion processes. Let $X(t)$ be a one dimensional diffusion process on an interval $(a, b)(-\infty \leq a<b \leq \infty)$ so that the Euclidean coordinate is the canonical scale (i.e. $X(t)$ in the Euclidean coordinate is a local martingale). Then, this process is determined by its speed measure $d m(x)$ and the Feller's boundary condition. Under this situation (assuming the support of $d m$ be $(a, b)$ ), there is a heat kernel $p_{t}(x, y)$ w.r.t. $d m$ such that

$$
-\lim _{t \rightarrow 0} 2 t \log p_{t}(x, y)=\left|\int_{x}^{y} \sqrt{\frac{1}{2} \frac{d m}{d x}(u) d u}\right|^{2} \forall x, y \in(a, b),
$$

where $\frac{d m}{d x}$ is a Radon-Nikodym derivative of the absolute continuous part of $d m$ w.r.t. Lebesgue measure $d x$ (this result is originally due to [14]). This

Communicated by Y. Takahashi, April 24, 1996.

1991 Math. Subject Classification(s) : 60F10, 60J60, 60J80

${ }^{*}$ Graduate School of Polymathematics, Nagoya University, Chikusa-ku, Nagoya 464-01, Japan 
type of short time asymptotic estimate is sometimes called Varadhan type estimate in honour of his celebrated work [19]. His philosophy is that taking this kind of limit, the intrinsic metric appears. In this case, the intrinsic metric is $d(x, y)=\left|\int_{x}^{y} \sqrt{\frac{1}{2} \frac{d m}{d x}(u) d u}\right|$. This result is deeply connected to the following large deviation. Let $P_{x}^{\epsilon}$ be the law for $X^{x}(\epsilon t)$ where $X^{x}$ is the process starting from $x$. For fixed $T>0$, set $C_{x}([0, T] \rightarrow E)=\{\phi \in C([0, T] \rightarrow E)$ : $\phi(0)=x\}$ with uniformly continuous topology where $E=[a, b]$ and $x \in(a, b)$. Define an $I$-function as

$$
I_{x}(\phi)=\left\{\begin{aligned}
\frac{1}{4} \int_{0}^{T} \frac{d m}{d x}(\phi(t))\left|\phi^{\prime}(t)\right|^{2} d t & \text { if } \phi \text { is absolute continuous } \\
\infty & \text { otherwise. }
\end{aligned}\right.
$$

Then, for each $A \subset C_{x}([0, T] \rightarrow E)$,

$$
-\inf _{\phi \in \operatorname{Int} A} I_{x}(\phi) \leq \liminf \epsilon \log P_{\epsilon \rightarrow 0}^{\epsilon}(A) \leq \lim \sup _{\epsilon \rightarrow 0} \epsilon \log P_{x}^{\epsilon}(A) \leq-\inf _{\phi \in \mathrm{CI} A} I_{x}(\phi) .
$$

We call this estimate Shilder type large deviation as this was firstly obtained by him on the Wiener measure ([18]).

S. Watanabe ([21]) further mentions that these estimates give no information (just $-\log p_{t}(x, y)=o(1 / t)$ as $\left.t \rightarrow 0\right)$ when $d m$ is singular and introduces one special example due to T. Fujita $([8])$ for which $d m$ is singular but detailed estimates can be obtained. Let $a=0, b=1, d m(x)=d F_{p}(x)$ where the continuous function $F_{p}:[0,1] \rightarrow[0,1](0<p<1)$ is defined as follows :

$$
\begin{aligned}
& F_{p}(0)=0, F_{p}(1)=1, \\
& F_{p}(x)=\left\{\begin{array}{rr}
(1-p) F_{p}(2 x) & \text { if } 0 \leq x \leq 1 / 2 \\
(1-p)+p F_{p}(2 x-1) & \text { if } 1 / 2 \leq x \leq 1 .
\end{array}\right.
\end{aligned}
$$

This $F_{p}(x)$, which is $x$ when $p=1 / 2$ and otherwise singular $\left(\right.$ i.e. $\frac{d F_{p}}{d x}(x)$ $=0$ a.e. $)$, is called de Rham function. Now, fix $0<p<1$ for which $\log (p / 2) / \log$ $((1-p) / 2) \notin \mathbf{Q}$ and set $s>0$ so that $\left(\frac{p}{2}\right)^{\frac{s}{1+s}}+\left(\frac{1-p}{2}\right)^{\frac{s}{1+s}}=1$. Setting $\bar{p}=$ $\left(\frac{p}{2}\right)^{\frac{s}{1+s}}$, the following holds.

Theorem 1.1. There exists a heat kernel $p_{t}(x, y)$ w.r.t. $d m$ and a positive continuous slowly varying function $L(t)$ (i.e. $\lim _{t \rightarrow 0} L(c t) / L(t)=1$ for all $c>$ $0)$ such that the following estimates hold.

1) $-\lim _{t \rightarrow 0} t^{s} L(t) \log p_{t}(x, y)=\left|F_{\bar{p}}(x)-F_{\bar{p}}(y)\right|^{1+s} \quad \forall x, y \in(0,1)$. 
2) For $A \subset C_{x}([0, t] \rightarrow[0,1])$,

$$
\begin{aligned}
-\inf _{\phi \in \operatorname{Int} A} I_{x}(\phi) \leq \liminf _{\epsilon \rightarrow 0} \epsilon^{s} L(\epsilon) \log P_{x}^{\epsilon}(A) & \leq \limsup \epsilon_{\epsilon \rightarrow 0}^{s} L(\epsilon) \log P_{x}^{\epsilon}(A) \\
& \leq-\inf _{\phi \in \mathrm{Cl} A} I_{x}(\phi)
\end{aligned}
$$

where $I_{x}$ is defined by

$$
I_{x}(\phi)=\left\{\begin{array}{c}
\int_{0}^{T}\left|\frac{d}{d t} F_{\bar{p}}(\phi(t))\right|^{1+s} d t \quad \text { if } F_{\bar{p}}(\phi(t)) \text { is absolute continuous } \\
\infty \text { otherwise. }
\end{array}\right.
$$

We are motivated by the results and consider the problem on fractals. The fractals we consider is a subclass of affine nested fractals, a class of finitely ramified fractals studied in [7]. Typical examples, which we express the results here in the introduction, are shown in Figure 1.1 (Sierpinski gasket) and Figure 1.2. The based fractals $E$ are constructed by an iterative procedure from the figures. We give conductance on each triangles : for Figure 1.1, the conductance on each triangle is $5 / 3$, while for Figure $1.2, \rho_{1}$ on small triangles and $\rho_{2}$ on the large triangle where the ratio of $\rho_{1}, \rho_{2}>1$ are arbitrarily chosen. By another iterative procedure, we can construct a Dirichlet form on the fractal. The based measure is a Bernoulli measure such that the mass on each triangle is $1 / 3$ for Figure 1.1, while for Figure 1.2, $\mu_{1}$ on small triangles and $\mu_{2}$ on the large triangle where $\mu_{1}, \mu_{2}>0,9 \mu_{1}+\mu_{2}=1$. Then, one can obtain a heat kernel $p_{t}(x, y)$ of the corresponding diffusion with apriori estimate (Theorem 2.6). Our results are concerning the Varadhan type estimate and the Schilder type large deviation for the process. Let $d(x, y)$ be a shortest path metric constructed in Section 2 and $d_{w}$ be a so called random walk dimension which expresses the average diffusing speed of particles (i.e. $E^{x}[d(x, X(t))] / t^{1 / d_{w}}$ is bounded from above and below for all $0<t<\infty)$. Then our main theorem is the following.

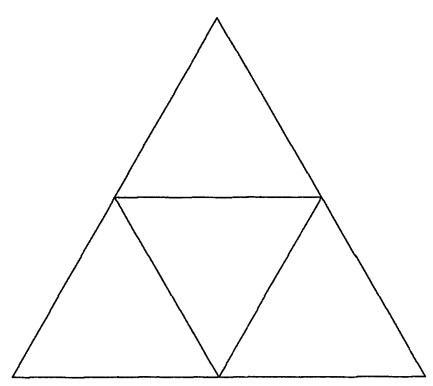

Figure 1.1

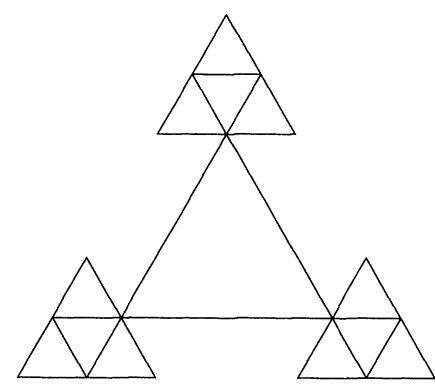

Figure 1.2 


\section{Theorem 1.2.}

a) For the case of Figure 1.1 with $\rho_{i}=5 / 3, \mu_{i}=1 / 3(1 \leq i \leq 3)$, there exists a non-constant positive continuous function $F(t)$ such that the following holds for all $z>0, x, y \in E$. (The right hand side is 0 when $d(x, y)=0$.)

$$
-\lim _{n \rightarrow \infty}\left((2 / 5)^{n} z\right)^{\frac{1}{d w-1}} \log p_{(2 / 5)^{n} z}(x, y)=d(x, y)^{\frac{d w}{d w-1}} F\left(\frac{z}{d(x, y)}\right) .
$$

b) For the case of Figure 1.2, if $\frac{\log \left(\rho_{1} / \mu_{1}\right)}{\log \left(\rho_{2} / \mu_{2}\right)} \notin \mathbf{Q}$, then the following holds.

1) $-\lim _{t \rightarrow 0} t^{1 /\left(d_{w-1}\right)} \log p_{t}(x, y)=d(x, y)^{\frac{d w}{d w-1}} \quad \forall x, y \in E$.

2) For $A \subset C_{x}([0, T] \rightarrow E)$,

$$
\begin{aligned}
-\inf _{\phi \in \operatorname{Int} A} I_{x}(\phi) \leq \liminf _{\epsilon \rightarrow 0} \epsilon^{\frac{1}{d w-1}} \log P_{x}^{\epsilon}(A) & \leq \lim \sup _{\epsilon \rightarrow 0} \epsilon^{\frac{1}{d w-1}} \log P_{x}^{\epsilon}(A) \\
& \leq-\inf _{\phi \in \mathrm{CI} A} I_{x}(\phi),
\end{aligned}
$$

where $I_{x}$ is defined by

$$
I_{x}(\phi)=\left\{\begin{aligned}
\int_{0}^{T}\left|\frac{d}{d t} d(x, \phi(t))\right|^{\frac{d w}{d w-1}} d t & \text { if } d(x, \phi(t)) \text { is absolute continuous } \\
\infty & \text { otherwise. }
\end{aligned}\right.
$$

As a corollary to Theorem $1.2 \mathrm{a}$ ), we see that Varadhan type estimate does not hold for the Sierpinski gasket (Corollary 4.1).

Using the idea of the proof, we can show that $L(t)$ in Theorem 1.1 can be chosen as a constant (Theorem 4.2).

In Section 2 we briefly explain the class of fractals we treat and explain how to construct the Dirichlet forms and the shortest path metrics. Section 3 is for the estimates of hitting times which is a key part of the proof of our results. We give the proof of Theorem 1.2 in Section 4.

The author thanks Dr. B. M. Hambly for fruitful discussions while he was visiting United Kingdom.

\section{§2. A Class of Affine Nested Fractals and Their Dirichlet Forms}

In [7], we defined a class of finitely ramified fractals called affine nested fractals and constructed Dirichlet forms for the fractals. Here we treat a subclass of the fractal and briefly summarize the construction.

Let $\alpha>1$, then an $\alpha$-similitude is a map $\Psi: \mathbf{R}^{D} \rightarrow \mathbf{R}^{D}$ such that

$$
\Psi(x)=\alpha^{-1} U(x)+a,
$$

where $U$ is a unitary, linear map and $a \in \mathbf{R}^{D}$. We will consider a finite family of $\alpha_{i}$-similitudes by $\left\{\Psi_{i}\right\}_{i=1}^{N}$. Without loss of generality, we assume $\Psi_{1}(x)=\alpha_{1}^{-1} x$. 
By Hutchinson [11], there exists unique non void compact set $E$ such that $E=$ $\bigcap_{i=1}^{N} \Psi_{i}(E)$ and this is a self-similar fractal. As each $\Psi_{i}$ is a contraction, it has a unique fixed point. Let $F$ be the set of fixed points of the $\Psi_{i}^{\prime}$ 's, $1 \leq i \leq N$. A point $x \in F$ is called an essential fixed point if there exist $i, j \in\{1, \cdots, N\}, i \neq$ $j$ and $y \in F$ such that $\Psi_{i}(x)=\Psi_{j}(y)$. We write $F^{(0)}$ for the set of essential fixed points. For $A \subset \mathbf{R}^{D}$, define $\Psi_{i_{1}, \cdots, i_{n}}(A)=\Psi_{i_{1}} \circ \cdots \circ \Psi_{i_{n}}(A)$. We will call the set $\Psi_{i_{1}, \cdots, i_{n}}\left(F^{(0)}\right)$ an $n$-cell and $\Psi_{i_{1}, \cdots, i_{n}}(E)$ an $n$-complex. Set

$$
F^{(n)}=\bigcup_{i_{1}, \cdots, i_{n}=1}^{N} \Psi_{i_{1}, \cdots, i_{n}}\left(F^{(0)}\right), F^{(\infty)}=\bigcup_{n=0}^{\infty} F^{(n)} .
$$

Taking closure, $E$ can be recovered : $E=C l\left(F^{(\infty)}\right)$.

We can now define an affine nested fractal as follows.

Definition 2.1. The set $E$ is an affine nested fractal if $\left\{\Psi_{1}, \cdots, \Psi_{N}\right\}$ satisfy :

(A1) (connectivity) For any 1 -cells $C$ and $C^{\prime}$, there is a sequence $\left\{C_{i}: i=0\right.$, $\cdots, n\}(n \in \mathbf{N})$ of $1-$ cells such that $C_{0}=C, C_{n}=C^{\prime}$ and $C_{i-1} \cap C_{i} \neq \phi, i=1, \cdots$, $n$.

(A2) (symmetry) If $x, y \in F^{(0)}$ then reflection in the hyperplane $H_{x y}=\{z: \mid z$ $-x|=| z-y \mid\}$ maps $F^{(n)}$ to itself.

(A3) (nesting) If $\left\{i_{1}, \cdots, i_{n}\right\},\left\{j_{1}, \cdots, j_{n}\right\}$ are distinct sequences then

$$
\Psi_{i_{1}, \cdots, i_{n}}(E) \cap \Psi_{j_{1}, \cdots, j_{n}}(E)=\Psi_{i_{1}, \cdots, i_{n}}\left(F^{(0)}\right) \bigcap \Psi_{j_{1}, \cdots, j_{n}}\left(F^{(0)}\right) .
$$

(A4) (open set condition) There is a non-empty, bounded open set $V$ such that the $\Psi_{i}(V)$ are disjoint and $\bigcup_{i=1}^{N} \Psi_{i}(V) \subset V$.

Our first further assumption is the following.

(A5) $\Psi_{i}(E) \cap \Psi_{j}(E)(i \neq j)$ is at most one point for $1 \leq i, j \leq N$.

(A6) For all $x, y, z \in F^{(0)}$ which are distinct, $|x-y|=|x-z|$.

Remark. 1) In [7], we thought we could deduce (A5) from (A1)-(A4) (Proposition 2.2), but the proof needed more assumption so that we add it as an assumption here (we do not know whether (A5) always holds for affine nested fractals or not).

2) (A6) implies that $F^{(0)}$ is a $D$-dimensional tetrahedron.

We next define a size equivalence class. The sets $\Psi_{i}(E)$ and $\Psi_{j}(E)$ are the same size if they can be mapped to each other by the composition of the reflection maps which appear in (A2). Let the number of 1-complexes with different size be $k_{0}$. We can order the 1-cells by their size and put a weight on each size : $\widetilde{\mathbf{r}}=\left(\widetilde{r}_{1}, \cdots, \widetilde{r}_{k_{0}}\right), \widetilde{r}_{i}>0\left(1 \leq i \leq k_{0}\right)$. (We use $\widetilde{r}$ when we distinguish cells by their size.) We call $(x, y) \in F^{(1)} \times F^{(1)}(x \neq y)$ size $i$ and write $\operatorname{Size}(x, y)$ 
$=i$ if $x, y$ are $F^{(1)}$-neighbours and the size of the 1-complex containing both $x$ and $y$ is $i$ (by (A5), there is a unique 1-complex containing both $x$ and $y$ ). Let the size of the 1-complex which contains an element of $F^{(0)}$ be $\widetilde{r}_{1}$. Also, let $r_{i}$ $=\tilde{r}_{\text {Size }\left(\Psi_{i}(E)\right)}$ where $\operatorname{Size}\left(\Psi_{i}(E)\right)$ is the size of $\Psi_{i}(E)(1 \leq i \leq N)$.

Now, we put conductance $r_{i}$ on $\Psi_{i}(E)$ and consider $F^{(1)}$ as a network. Then, for each $f \in l\left(F^{(1)}\right)=\left\{f: F^{(1)} \rightarrow \mathbf{R}\right\}$, the energy of this network is

$$
\overline{\mathscr{E}}_{1}(f, f)=\frac{1}{2} \sum_{x, y \in F^{(1)}}(f(x)-f(y))^{2} J_{x y}
$$

where $J_{x y}=1 / \tilde{r}_{\operatorname{Size}(x, y)}$ if $x, y$ are $F^{(1)}$-neighbours and $J_{x y}=0$ otherwise. We can then induce a network on $F^{(0)}$ by solving a variational problem ([1] e.t.c.) :

Proposition 2.2. There exists unique $\lambda>0$ such that

$$
\inf \left\{\overline{\mathscr{E}}_{1}(f, f):\left.f\right|_{F^{(0)}}=v\right\}=\frac{1}{2 \lambda} \sum_{x, y \in F^{(0)}}(f(x)-f(y))^{2} \text { for all } v \in l\left(F^{(0)}\right) \text {. }
$$

Set $\rho_{i}=\lambda / r_{i}$. We assume the following throughout this paper.

(B1) For all $1 \leq i \leq N, \rho_{i}>1$.

Remark that (B1) always holds if we take $\widetilde{r}_{i}=\widetilde{r}_{j}$ for all $1 \leq i \leq j \leq k_{0}$. Let $\mu$ be a Bernoulli measure on $E$ such that $\mu\left(\Psi_{i}(E)\right)=\mu_{i}>0\left(\sum_{i=1}^{N} \mu_{i}=1\right)$.

We can now define the Dirichlet form for the affine nested fractal. Let $f$, $g \in l\left(F^{(\infty)}\right)=\left\{f: F^{(\infty)} \rightarrow \mathbf{R}\right\}$ and define

$$
\begin{aligned}
\mathscr{E}_{n}(f, g)=\frac{1}{2} & \sum_{1 \leq k_{1} \cdots k_{n} \leq N} \sum_{x, y \in F(0)} \rho_{k_{1} \cdots k_{n}}\left(f\left(\Psi_{k_{1}, \cdots, k_{n}}(x)\right)-f\left(\Psi_{k_{1}, \cdots, k_{n}}(y)\right)\right) \\
& \times\left(g\left(\Psi_{k_{1}, \cdots, k_{n}}(x)\right)-g\left(\Psi_{k_{1}, \cdots, k_{n}}(y)\right)\right)
\end{aligned}
$$

where $\rho_{k_{1} \cdots k_{n}}=\rho_{k_{1}} \cdots \rho_{k_{n}}$. This is a energy of the network on $F^{(n)}$ with conductance $\rho_{k_{1} \cdots k_{n}}$ on $\Psi_{k_{1} \cdots k_{n}}(E)$. By Proposition 2.2,

$$
\mathscr{E}_{n}\left(\left.f\right|_{F^{(n)}},\left.f\right|_{F^{(n)}}\right) \leq \mathscr{E}_{n+1}\left(\left.f\right|_{F^{(n+1)}},\left.f\right|_{F^{(n+1)}}\right) \text { for all } f \in l\left(F^{(\infty)}\right) \text {. }
$$

For $f \in l\left(F^{(\infty)}\right)$, define $\mathscr{F}=\left\{f: \sup _{n} \mathscr{E}_{n}(f, f)<\infty\right\}$ and $\mathscr{E}(f, f)=\lim _{n \rightarrow \infty}$ $\mathscr{E}_{n}(f, f)$. Then, from $[10],[16]$ and for the more general class of P.C.F. self-similar sets in [12], we have the following.

Theorem 2.3. 1) Any function in $\mathcal{F}$ can be extended uniquely to a continuous function on $E$ (thus we can consider $\mathscr{F} \subset C(E)=\{f: f$ is a continuous function on $E\})$. Further, $(\mathscr{E}, \mathcal{F})$ is a local regular Dirichlet form on $\mathbf{L}^{2}(E, \mu)$ which has the following properties.

$$
\mathscr{E}(f, g)=\sum_{i=1}^{N} \rho_{i} \mathscr{E}\left(f \circ \Psi_{i}, g \circ \Psi_{i}\right) \text { for all } f, g \in \mathcal{F},
$$




$$
\sup _{x \in E}|f(x)| \leq c_{2.1} \cdot \mathscr{E}_{1}(f, f) \text { for all } f \in \mathscr{F},
$$

where $c_{2.1}>0$ and $\mathscr{E}_{\beta}(\cdot, \cdot)=\mathscr{E}(\cdot, \cdot)+\beta(\cdot, \cdot)_{\mathbf{L}^{2}(E, \mu)}$ for $\beta>0$.

2) $\mathscr{E}_{\beta}$ admits a positive symmetric continuous reproducing kernel $g_{\beta}(\cdot, \cdot)$ which are uniformly bounded and equi-uniform continuous w.r.t. $\beta>0$ on $E$. The corresponding diffusion is point recurrent.

Note that the based metric in the statement 2) will be introduced later. Let $\tau_{i}=\rho_{i} / \mu_{i} . \quad \tau_{i}$ is a time scale for the process on $\Psi_{i}(E)$ (i.e. the average time for a particle to cross $\Psi_{i}(E)$ is $\tau_{i}^{-1}$ times the average time to cross $E$ ).

We next introduce an intrinsic metric for the Dirichlet forms which we call a shortest path metric. For $x, y \in F^{(m)}$, let

(6) $\pi_{m}(x, y)=\left\{\pi_{m}: \pi_{m}\right.$ is an $m$-walk in $E$ from $x$ to $y$ which does not contain multiple points\}.

Here $\pi_{m}=\left\{p_{k}, p_{k+1}\right\}_{k=1}^{l}$ is called an $m$-walk if $l \in \mathbf{N}, p_{k} \in F^{(m)}$ for $1 \leq k \leq l$, $p_{k}$ and $p_{k+1}$ join in the same $m$-cell for $1 \leq k \leq l-1$. For $\pi_{m}=\left\{p_{k}, p_{k+1}\right\}_{k=1}^{l} \in$ $\pi_{m}(x, y)$, we say the length of $\pi_{m}$ is $l$ and denote it by $\left|\pi_{m}\right|=l$. For $\pi \in \pi_{1}(x$, $y$ ), let $\mathbf{v}(\pi)$ be an $k_{0}$-dimensional vector such that $(\mathbf{v}(\pi))_{k}$ is the number of $k$-size steps in the path $\pi\left(1 \leq k \leq k_{0}\right)$. We assume the following.

(A7) For $x, y \in F^{(0)}, x \neq y$, there exists a path $\bar{\pi} \in \pi_{1}(x, y)$ such that $(\mathbf{v}(\pi))_{k} \geq$ $(\mathbf{v}(\tilde{\pi}))_{k}\left(1 \leq k \leq k_{0}\right)$ for all $\pi \in \pi_{1}$.

In the following, we fix the shortest $F^{(1)}$-path from 0 to $x_{0} \in F^{(0)} \backslash\{0\}\left(x_{0}\right.$ is arbitrarily fixed) and denote it $\hat{\pi}=\left\{\left(p_{k}^{0}, p_{k+1}^{0}\right)\right\}_{k=1}^{|\vec{\pi}|}$. Set $\gamma>0$ so that $\sum_{k=1}^{|\hat{\pi}|} \tau_{p_{k, j}^{0}, p_{k+1}^{0}}^{-\gamma}$ $=1$. Now we define the distance on $F^{(m)}$ as follows :

$$
d_{F(m)}(x, y)=\min _{\left.\pi \in \pi_{\pi}(x, y), \pi=\left\{\left(p_{k}, p_{k+1}\right)\right\}\right\}_{k=1}} \sum_{k=1}^{|\pi|} \tau_{p_{k}, p_{k+1}}^{-\gamma}
$$

Here $\tau_{p, q}=\tau_{i_{1} \cdots i_{m}}$ if the $m$-complex containing both $p$ and $q$ is $\Psi_{i_{1} \cdots i_{m}}(E)$.

Lemma 2.4. ([7]) 1) $d_{F^{(m)}}(x, y)=d_{F^{(m+1)}}(x, y)$ if $x, y \in F^{(m)}$.

2) For any choice of $p, q \in E$, define $d(p, q)$ by

$$
d(p, q)=\lim _{n \rightarrow \infty} d\left(p_{n}, q_{n}\right)
$$

where $p_{n}, q_{n} \in F^{(\infty)}$ and $p_{n} \rightarrow p, q_{n} \rightarrow q$ as $n \rightarrow \infty$. Then $d$ is well defined and $d$ is a metric on $E$.

For the diffusion $X(t)$ corresponding to the Dirichlet form $E$, let

$$
W_{n}=\inf \left\{t \geq 0: X(t) \in F^{(n)} \backslash\{X(0)\}\right\} .
$$

Setting $\phi(s)=E^{0}\left[\exp \left(-s W_{0}\right)\right]$, we have the following. 
Proposition 2.5. 1) There exist positive constants $c_{2.2}, c_{2.3}$ such that

$$
c_{2.2} \leq \phi(s) / \prod_{k=1}^{|\hat{\pi}|} E^{p_{k}^{0}}\left(\exp \left(-s W_{1}\right) \mid X\left(W_{1}\right)=p_{k+1}^{0}\right) \leq c_{2.3} .
$$

2) There exist positive constants $c_{2.4}, c_{2.5}, c_{2.6}, c_{2.7}$ such that

$$
c_{2.4} \exp \left(-c_{2.5} s^{1 / d w}\right) \leq \phi(s) \leq c_{2.6} \exp \left(-c_{2.7} s^{1 / d w}\right) \text { for all } s \geq 0,
$$

where $d_{w}=1 / \gamma$.

Proof. By the symmetry and strong Markov property,

$$
\phi(s)=\sum_{\substack{\left.\pi \in \pi_{1}\left(0, x_{0}\right),\right\}_{k} \\ \pi=\left\{p_{k}, p_{k+1}\right\}_{k=1}}} P^{x}[\pi] \cdot \prod_{k=1}^{|\pi|} E^{p_{k}}\left[\exp \left(-s W_{1}\right) \mid X\left(W_{1}\right)=p_{k+1}\right]
$$

On the other hand, it is easy to prove that, if $\tau_{p_{k, p}^{o} p_{k+1}^{o}}=\tau_{p_{s}, p_{s+1}}$, then $c_{2.8} \leq E^{p_{j}}\left[\exp \left(-s W_{1}\right) \mid X\left(W_{1}\right)=p_{j+1}\right] / E^{p_{k}^{0}}\left[\exp \left(-s W_{1}\right) \mid X\left(W_{1}\right)=p_{k+1}^{0}\right] \leq c_{2.9}$

for some $c_{2.8}, c_{2.9}>0$. (Much stronger fact will be proved in Lemma 3.1.) Now, using the assumption (A7), we can factorize the right hand side of (9) and obtain 1). 2) is proved in [7] Proposition 5.2 (we did not mention lower bound there, but that can be proved in the same way as [15] Proposition 3.2).

For the heat kernel, we have the following estimates essentially proved in [7]. Remark that changing the based Bernoulli measure corresponds to a singular time change for the process and changing the measure also changes the metric.

Theorem 2.6. There exists a jointly continuous transition density $p_{t}(x, y)$ for the semigroup on $\mathbf{L}^{2}(E, \mu)$ which satisfies the following.

$$
\begin{aligned}
& \min _{0 \leq s \leq 1} p_{s}(x, x)>0 \text { for all } x \in E, \\
& p_{t}(x, y) \leq c_{2.10} t^{-1} \exp \left(-c_{2.11} \Psi(d(x, y), t)\right)
\end{aligned}
$$

for all $0<t<1, x, y \in E$, where $\Psi(z, t)=\left(z^{d w} t^{-1}\right)^{1 /\left(d_{w}-1\right)}$ and $c_{2.10}, c_{2,11}>0$.

Proof. (10) can be proved in the same way as [2] Lemma 7.1. The diagonal estimate of (11) comes from the facts that $g_{\beta}$ is uniformly bounded, $p_{t}(x, x)$ is non-increasing w.r.t. $t$ and the expression $g_{\beta}(x, x)=\int_{0}^{\infty} \exp (-\beta t) p_{t}(x$, $x) d t$. The proof of the off-diagonal estimate is the same as Theorem 5.7 in [7]. 
Remark. 1) In [7], we consider unbounded affine nested fractals and their Dirichlet forms. Let $E^{\langle n\rangle}=\alpha_{1}^{n} E$ and $\widehat{E}=\bigcup_{n=1}^{\infty} E^{\langle n\rangle} . \widehat{E}$ is the unbounded affine nested fractal. Also, let $\widetilde{\mu}$ be the Bernoulli measure on $\widehat{E}$ such that

$$
\widetilde{\mu}(A)=\mu_{1}^{-n} \mu \underbrace{\left(\Psi_{1} \circ \cdots \circ \Psi_{1}\right.}_{n \text { times }}(A)) \text { for all } A \subset E^{\langle n\rangle}, n \in \mathbf{N} .
$$

Then, by the similar way, we can construct a local regular Dirichlet form on $\mathbf{L}^{2}(\hat{E}, \widetilde{\mu})$. The main theorem in [7] was about the Aronson type estimates for the transition density $\bar{D}_{t}(x, y)$ of the form with respect to a special measure. Here we briefly state the result. Let $S$ be the unique constant which satisfies $\sum_{i=1}^{N} \rho_{i}^{-s}=1$. Then, for the Bernoulli measure $\tilde{\mu}$ satisfying $\mu_{i}=\rho_{i}^{-s}$ for $1 \leq i \leq N$, there exist positive constants $c_{2.12}, c_{2.13}, c_{2.14}, c_{2.15}$ such that

$$
\begin{aligned}
& c_{2.12} t^{-\frac{d_{s}}{2}} \exp \left(-c_{2.13} \Psi(d(x, y), t)\right) \\
& \quad \leq \bar{p}_{t}(x, y) \leq c_{2.14} t^{-\frac{d_{s}}{2}} \exp \left(-c_{2.15} \Psi(d(x, y), t)\right)
\end{aligned}
$$

for all $0<t<\infty, x, y \in \widehat{E}$. Our question is whether more precise estimates will be possible in very short time.

2) The results introduced in this section, except Proposition 2.51 ), is valid without assuming (A6), (A7). Also, remark that the fractals in Figure 1.1 and Figure 1.2 satisfy $(\mathrm{A} 1) \sim(\mathrm{A} 7)$.

In the following of this paper, we assume $(\mathrm{A} 1) \sim(\mathrm{A} 7)$ and $(\mathrm{B} 1)$.

\section{§3. Estimates of Hitting Times}

In this section, we will have sharp estimates of hitting times. First, we define a collection of words, $\Lambda_{n} \subset \cup_{m \geq 1}\{1, \cdots, N\}^{m}$, by

$$
\Lambda_{n}=\left\{\omega=\omega_{1} \cdots \omega_{l} \in \bigcup_{m \geq 1}\{1, \cdots, N\}^{m}: \tau_{\omega_{1} \cdots \omega_{l-1}}<\tau_{i_{0}}^{n} \leq \tau_{\omega_{1} \cdots \omega_{l}}\right\} .
$$

Here $\tau_{i_{0}}=\max _{i} \tau_{i}$ and we set $\tau_{\omega_{1} \cdots \omega_{l-1}}=1$ when $l=1$. This set was introduced in [13] and similar set was considered in [7]. Define $H^{\left(\Lambda_{n}\right)}=$ $\bigcup_{\omega \in \Lambda_{n}} \Psi_{\omega}\left(F^{(0)}\right)$. We call $\Lambda_{n}$-cells, $\Lambda_{n}$-complexes in the same way as we did for $F^{(n)}$. Then the time scale of $\Lambda_{n}$-complexes differs at most $\tau_{i_{0}}$. For $H^{\left(\Lambda_{n}\right)}$ neighbours $p, q$, set $\tau_{p, q}=\tau_{\omega_{1} \cdots \omega_{l}}$ if $p, q \in \Psi_{\omega_{1} \cdots \omega_{l}}\left(F^{(0)}\right)$.

Lemma 3.1. There exist $c_{3.1}, c_{3.2}>0$ such that

$$
c_{3.1} \phi\left(s / \tau_{p, q}\right) \leq E^{p}\left(\exp \left(-s W_{\Lambda_{n}}\right) \mid X\left(W_{\Lambda_{n}}\right)=q\right) \leq c_{3.2} \phi\left(s / \tau_{p, q}\right)
$$

for all $s \geq 0, p, q \in H^{\left(\Lambda_{n}\right)}$ which are $H^{\left(\Lambda_{n}\right)}$-neighbours.

Here $W_{A_{n}}=\inf \left\{t \geq 0: X(t) \in H^{\left(\Lambda_{n}\right)} \backslash\{X(0)\}\right.$. 
Proof. First, remark that from (A6), we have

$$
E^{0}\left[\exp \left(-s \tau_{p, q}^{-1} W_{0}\right)\right]=E^{0}\left[\exp \left(-s \tau_{p, q}^{-1} W_{0}\right) \mid X\left(W_{0}\right)=x_{0}\right]
$$

for an arbitrary $x_{0} \in F^{(0)} \backslash\{0\}$. Now pick up all $\Lambda_{n}$-cells which intersects $p$ and extend each cells (except the cell containing $q$ ) $\alpha_{1}^{l}$ times ( $l$ is the smallest integer satisfying $\tau_{1}^{l} \geq \tau_{i_{0}}$ ). Let $\bar{W}$ be the first hitting time to the boundaries (except $p$ ) of these extended cells. (Giving conductance and measure similarly to that in Section 2, one can construct Dirichlet forms on this space.) In the same way, shrink each cells (except the cell containing $q$ ) $\alpha_{1}^{-l}$ times and let $\underline{W}$ be the first hitting time to the boundaries (except $p$ ) (see Figure 3.1). By the construction, the average speeds of particles crossing in the extended (shrunk) complexes are bigger (smaller) than that in the complex containing $p$ and $q$. Also, as the number of complexes containing $p$ is bounded from above by some positive constant (independent of the choice of $p$ ), $E^{0}\left[\exp \left(-s \tau_{p, q}^{-1} W_{0}\right) \mid X\left(W_{0}\right)=x_{0}\right] /$ $E^{p}\left[\exp \left(-s W_{A_{n}}\right) \mid X\left(W_{A_{n}}\right)=q\right]$ is uniformly bounded from above and below by some positive constants. Therefore we can show

$$
\begin{aligned}
c_{3.3} E^{p}[\exp (-s \bar{W}) \mid X(\bar{W})=q] & \leq E^{0}\left[\exp \left(-s \tau_{p, q}^{-1} W_{0}\right) \mid X\left(W_{0}\right)=x_{0}\right] \\
& \leq c_{3.4} E^{p}[\exp (-s \underline{W}) \mid X(\underline{W})=q] .
\end{aligned}
$$

Now

$$
\begin{aligned}
E^{p}\left(\exp \left(-s W_{A_{n}}\right) \mid X\left(W_{A_{n}}\right)=q\right) & \leq \frac{P^{p}(X(\bar{W})=q)}{P^{p}\left(X\left(W_{A_{n}}\right)=q\right)} E^{p}(\exp (-s \bar{W}) \mid X(\bar{W})=q) \\
& \leq \frac{1}{c_{3.3}} \frac{P^{p}(X(\bar{W})=q)}{P^{p}\left(X\left(W_{A_{n}}\right)=q\right)} \phi\left(s / \tau_{p, q}\right)
\end{aligned}
$$

where the first inequality is from $\left\{X\left(W_{A_{n}}\right)=q\right\} \subset\{X(\bar{W})=q\}$ and the second is from (12) and (13). As $P^{p}(X(\bar{W})=q) / P^{p}\left(X\left(W_{A_{n}}\right)=q\right)$ is uniformly bounded from above, we obtain the second inequality. The first inequality can be obtained in the same way.

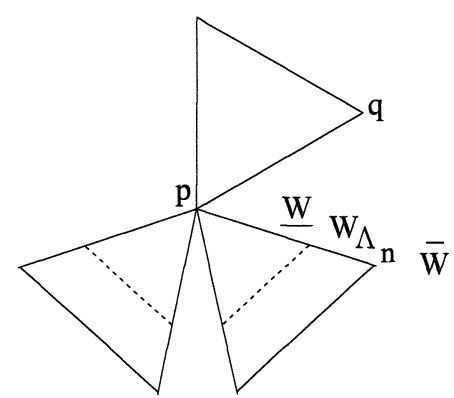

Figure 3.1 
For the proof of the next key lemma, we use the following version of the well-known renewal theorem. This version of the renewal theorem is used in [13] to show the asymptotic behaviour of eigenvalues of Laplacians on P.C.F. self-similar fractals.

Theorem 3.2 (The Renewal Theorem). Let $\nu$ be a Borel probability measure on $[0, \infty)$ such that $\int_{0}^{\infty} x \nu(d x)<\infty$. Let $u \in \mathbf{L}^{1}(\mathbf{R})$ be such that $u(x) \rightarrow 0$ as $|x| \rightarrow \infty$. Suppose that $z$ is a bounded measurable function which satisfies the renewal equation

$$
z(x)=u(x)+\int_{0}^{\infty} z(x-t) \nu(d t), \text { for } x \in \mathbf{R},
$$

and such that $z(x) \rightarrow 0$ as $x \rightarrow-\infty$. Then

1) Non-Lattice Case: If the support of $\nu$ does not lie in any discrete subgroup of $\mathbf{R}$, then the limit $z(\infty)=\lim _{x \rightarrow \infty} z(x)$ exists and

$$
z(\infty)=\left(\int_{0}^{\infty} x \nu(d x)\right)^{-1} \int_{-\infty}^{\infty} u(x) d x
$$

2) Lattice Case: If the support of $\nu$ lies in some discrete subgroup of $\mathbf{R}$, then if $T$ is the greatest common divisor of the support of $\nu$, the limit $G(t)=$ $\lim _{n \rightarrow \infty} z(t+n T)$ exists for every $t$ and

$$
G(t)=\left(\int_{0}^{\infty} x \nu(d x)\right)^{-1} \sum_{j=-\infty}^{\infty} u(t+j T) .
$$

Lemma 3.3. Let $L(s)=-s^{-1 / d w} \log \phi(s)$.

1) Non-Lattice Case: If the group $\sum_{k=1}^{k_{0}} \mathbf{Z} \log \tau_{k}$ is a dense subgroup of $\mathbf{R}$, then the limit $\lim _{s \rightarrow \infty} L(s)$ exists and it is positive.

2) Lattice Case : If $\sum_{k=1}^{k_{0}} \mathbf{Z} \log \tau_{k}$ is a discrete subgroup of $\mathbf{R}$, let $\log h$ be its positive generator. Then the limit $k(s)=\lim _{n \rightarrow \infty} L\left(s \cdot h^{n}\right)$ exists and it is positive.

Proof. Let

$$
\begin{aligned}
u(x)=-\exp \left(-x / d_{w}\right) & \left(\log \left(\frac{\phi(\exp x) \wedge(1-\epsilon)}{1-\epsilon}\right)\right. \\
& \left.-\sum_{k=1}^{|\hat{\pi}|} \log \left(\frac{\phi\left(\exp \left(x / a_{k}\right)\right) \wedge(1-\epsilon)}{1-\epsilon}\right)\right)
\end{aligned}
$$

where $\hat{\pi}=\left\{\left(p_{k}^{0}, p_{k+1}^{0}\right)\right\}_{k=1}^{\langle\hat{\pi}|}$ is the shortest $F^{(1)}$-path introduced in Section 2 and $a_{k}$ $=\tau_{p_{k, p_{k+1}^{\circ}}^{0}}$. By the truncation, we see $u(x)=0$ for all $x \leq-N$ when $N$ is large enough. Also, by (7) and Lemma 3.1, we see

$$
|u(x)| \leq c_{3.5} \exp \left(-x / d_{w}\right) \text { for all } x \geq 0
$$

so that $u(x) \rightarrow 0$ as $x \rightarrow \infty$. By these facts, $u \in \mathbf{L}^{1}(\mathbf{R})$. Set 


$$
\begin{gathered}
z(x)=-\exp \left(-x / d_{w}\right) \log \left(\frac{\phi(\exp x) \wedge(1-\epsilon)}{1-\epsilon}\right), \\
\nu(d t)=\sum_{k=1}^{|\tilde{\mu}|} a_{k}^{-1 / d_{w}} \delta_{\log a_{k}}(d t) .
\end{gathered}
$$

By the truncation, $z(x) \rightarrow 0$ as $x \rightarrow-\infty$ and by (8), $z(x)$ is bounded. $\nu$ is a probability measure such that $\int_{0}^{\infty} x \nu(d x)=\sum_{k} a_{k}^{-1 / d w} \log a_{k}<\infty$ and they satisfy (14). Thus by the renewal theorem mentioned above, we obtain the results. Note that the positivity of the limits comes from (8).

The next lemma relates hitting times to distances.

Lemma 3.4. For the case of the Sierpinski gasket with $\rho_{i}=5 / 3, \mu_{i}=1 / 3$ $(1 \leq i \leq 3)$ or for the non-lattice case, the following holds.

$$
\lim _{s \rightarrow \infty} \frac{-1}{L(s) s^{1 / d_{w}}} \log E^{x}\left[\exp \left(-s \tau_{y}\right)\right]=d(x, y) \quad \forall x, y \in E,
$$

where $\tau_{y}=\inf \{t \geq 0: X(t)=y\}$. This convergence is compact uniform w.r.t. d.

Proof. Non-lattice case : First, let us consider the case $x, y \in F^{(\infty)}$. In this case, we can choose $m$ (depending on $x$ and $y$ ) such that $x, y \in H^{(\Lambda m)}$. Taking a shortest $H^{(\Lambda m)}$-path $\tilde{\pi}=\left\{x_{0}, \cdots, x_{n}\right\}$ connecting $x\left(=x_{0}\right)$ and $y\left(=x_{n}\right)$, we see $d(x, y)=\sum_{i=0}^{n-1} d\left(x_{i}, x_{i+1}\right)$ by the geodesic property of this metric. On the other hand,

$$
\begin{aligned}
& E^{x}\left[\exp \left(-s \tau_{y}\right)\right]=\sum_{\pi \in \pi_{H^{\operatorname{un} m}}(x, y)} E^{x}\left[\exp \left(-s \tau_{y}\right) \mid \pi\right] P(\pi)
\end{aligned}
$$

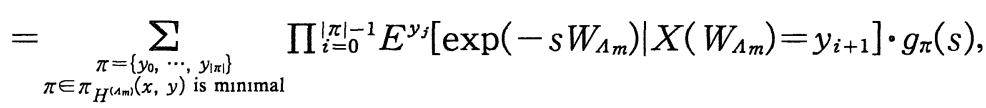

for some $g_{\pi}$ bounded from above and below $\left(\pi_{H^{(\Delta, m)}}(x, y)\right.$ is defined in the same way as (6)). The third equation is a factorization of the second by each minimal path (remark that $\tilde{\pi}$ is one of the minimal paths). Using Lemma 3.1, we have

$$
\begin{aligned}
c_{3.6} \prod_{i=0}^{n-1} \phi\left(s / \tau_{x_{i}, x_{i+1}}\right) & \leq E^{x}\left[\exp \left(-s \tau_{y}\right)\right] \\
& \leq c_{3.7} \prod_{i=0}^{n-1} \phi\left(s / \tau_{x_{i}, x_{i+1}}\right)\left\{\sum_{\pi} \frac{\prod_{\pi} \phi\left(s / \tau_{y_{i}, y_{i+1}}\right)}{\prod_{\pi} \phi\left(s / \tau_{x_{i}, x_{i+1}}\right)} \cdot g_{\pi}(s)\right\} \\
& \equiv c_{3.7} \prod_{i=0}^{n-1} \phi\left(s / \tau_{x_{i}, x_{i+1}}\right)\left\{h_{\pi}(s)\right\} .
\end{aligned}
$$

Taking $\log$ and divided by $\log \phi(s)$, we have $\frac{\log c_{3.6}}{\log \phi(s)}+\sum \frac{\log \phi\left(s / \tau_{x_{i}, x_{i+1}}\right)}{\log \phi(s)} \geq \frac{\log E^{x}\left[\exp \left(-s \tau_{y}\right)\right]}{\log \phi(s)}$ 


$$
\geq \frac{\log c_{3.7}}{\log \phi(s)}+\sum \frac{\log \phi\left(s / \tau_{x_{i}, x_{i+1}}\right)}{\log \phi(s)}+\frac{\log h_{\pi}(s)}{\log \phi(s)}
$$

Taking $s \rightarrow \infty$,

$$
\frac{\log \phi\left(s / \tau_{x_{i}, x_{i+1}}\right)}{\log \phi(s)}=\frac{\tau_{x_{i}, x_{i+1}}^{-1 / d_{w}} L\left(s / \tau_{x_{i}, x_{i+1}}\right)}{L(s)} \rightarrow \tau_{x_{i}, x_{i+1}}^{-1 / d w}=d\left(x_{i}, x_{i+1}\right)
$$

as $\lim _{s \rightarrow \infty} L(s)$ exists. On the other hand, by Lemma 3.3, we can take $c_{2.5}-c_{2.7}$ $\rightarrow 0$ in (8) as $s \rightarrow \infty$. By simple estimates using this fact, we have $\log h_{\pi}(s) / \log$ $\phi(s) \rightarrow 0$ as $s \rightarrow \infty$. As $\phi(s) \rightarrow 0, \log c_{3.6(3.7)} / \log \phi(s) \rightarrow 0$. Thus,

$$
\lim _{s \rightarrow \infty} \frac{-\log E^{x}\left[\exp \left(-s \tau_{y}\right)\right]}{L(s) s^{1 / d w}}=\lim _{s \rightarrow \infty} \frac{\log E^{x}\left[\exp \left(-s \tau_{y}\right)\right]}{\log \phi(s)}=\sum_{i} d\left(x_{i}, x_{i+1}\right)=d(x, y) .
$$

Now, for $x, y \in E$, take sequences $x_{n}, y_{n} \in F^{(\infty)}$ so that $x_{n} \rightarrow x, y_{n} \rightarrow y$. We have already proved

$$
\lim _{s \rightarrow \infty} \frac{\log E^{x_{n}}\left[\exp \left(-s \tau_{y_{n}}\right)\right]}{\log \phi(s)}=d\left(x_{n}, y_{n}\right) .
$$

Noting $E^{x_{n}}\left[\exp \left(-s \tau_{y_{n}}\right)\right]=g_{s}\left(x_{n}, y_{n}\right) / g_{s}\left(x_{n}, x_{n}\right)$ and equi-uniform continuity of the reproducing kernel (Theorem 2.32 )), we obtain (15) for $x, y \in E$. Using the equi-uniform continuity of the reproducing kernel again, it is easy to show that this convergence is compact uniform.

Sierpinski gasket with $\rho_{i}=5 / 3, \mu_{i}=1 / 3$ : In this case, $W_{0}$ is a limit random variable of a supercritical branching process divided by its mean and

$$
\phi(5 z)=\frac{\phi(z)^{2}}{4-3 \phi(z)}
$$

(see [3] Section 2). Using this fact, arguments are simpler. For the case $x, y$ $\in F^{(\infty)}$, choose $n$ so that $x, y \in H^{\left(\Lambda_{m}\right)}\left(=F^{(m)}\right.$ in this case), let a shortest $F^{(m)}$ path be $\tilde{\pi}=\left\{x_{0}, \cdots, x_{n}\right\}$ connecting $x\left(=x_{0}\right)$ and $y\left(=x_{n}\right)$. Then,

$$
\begin{aligned}
E^{x}\left[\exp \left(-s \tau_{y}\right): X\left(W^{k-1}\right)=x_{k}, 1 \leq k \leq n\right] & \leq E^{x}\left[\exp \left(-s \tau_{y}\right)\right] \\
& \leq E^{0}\left[\exp \left(-s \sum_{i} W^{i}\right)\right]
\end{aligned}
$$

where $W^{0}=W_{m} \stackrel{\mathrm{d}}{=} W_{0} / 5^{m}, W^{i}=\inf \left\{t>W^{i-1}: X(t) \in F^{(m)} \backslash\left\{X\left(W^{i-1}\right)\right\}\right\}$. The left equation is bounded from below by $(1 / 4)^{n} \phi\left(s / 5^{m}\right)^{n}$ and the right equation equals $\phi\left(s / 5^{m}\right)^{n}$. Further, by (16), $\log \phi\left(s / 5^{m}\right)=\frac{1}{2^{m}} \log \phi(s)+O(1)$. Thus, we have

$$
\lim _{s \rightarrow \infty} \frac{\log E^{x}\left[\exp \left(-s \tau_{y}\right)\right]}{\log \phi(s)}=n / 2^{m}=d(x, y)
$$

For the general $x, y \in E$, we have the result by the same proof as that of 
non-lattice case.

\section{§4. Proof of Theorem $\mathbf{1 . 2}$}

We first treat the Sierpinski gasket with $\rho_{i}=5 / 3, \mu_{i}=1 / 3(1 \leq i \leq 3)$.

Proof of Theorem $1.2 a$ ). For $x=y$, the result is obvious from Theorem 2.6. So we assume $x \neq y$. First, by Lemma 3.3, we see $k(s)=\lim _{n \rightarrow \infty} L\left(s^{\circ} 5^{n}\right)>$ 0 . As we remarked, $W_{0}$ is a limit random variable of a supercritical branching process divided by its mean in this case. Further, It is shown in [3] Section 3 that this $k(s)$ is not a constant (see also [4]). Now, for fixed $x, y \in E$, set $\bar{k}(s)$ $=-d(x, y) s^{1 / d w} k(s)$. By a simple calculation using (16), we see that $\bar{k}(s)$ is convex and real analytic on $\mathbb{R}_{+}$(in fact, these are properties of the so called Böttcher function composed with a Laplace transform of a limit random variable of some branching process (see [5])). Taking a subsequence in Lemma 3.4 ,

$$
\lim _{n \rightarrow \infty} 5^{-n / d w} \log E^{x}\left[\exp \left(-5^{n} s \tau_{y}\right)\right]=\bar{k}(s) .
$$

Let $a_{n}=5^{n / d w}, Y_{n}=-5^{n} \tau_{y}$. Using a variant of the Gärtner-Ellis theorem introduced in Section 2 of [5], we have for each $t<0$

$$
-\lim _{n \rightarrow \infty} \frac{1}{a_{n}} \log P\left(Y_{n} \geq a_{n} t\right)=\bar{k}^{*}(t)
$$

where $\bar{k}^{*}(t)=\sup _{s}\{t s-\bar{k}(s)\}$ (remark that $\bar{k}(s) \leq 0$ ). By simple calculations, we obtain

$$
\begin{aligned}
-\lim _{n \rightarrow \infty}\left(\left(\frac{2}{5}\right)^{n} z\right)^{\frac{1}{d_{w}-1}} \log P^{x}\left(\tau_{y} \leq\left(\frac{2}{5}\right)^{n} z\right) & =d(x, y)^{\frac{d_{w}}{d_{w}-1}} F\left(\frac{z}{d(x, y)}\right) \\
& \left(=z^{\frac{1}{d_{w-1}}} \bar{k}^{*}(-z)\right),
\end{aligned}
$$

where $F(y)=y^{1 /\left(d_{w}-1\right)} \sup _{s}\left\{k(s) s^{1 / d w}-y s\right\}$. It is easy to check that $F(y)>0$ for all $y \in \mathbb{R}_{+}$. Also, we can easily check that $F$ is constant if $k$ is. Thus, by a one-to-one correspondence of Legendre transform, we see $F$ is not constant as $k$ is not. Noting that $p_{t}(x, y)=\int_{0}^{t} p_{t-s}(y, y) P^{x}\left(\tau_{y} \in d s\right)$ and $A \equiv \min _{0 \leq s \leq t} p_{t-s}(y, y)$ $>0$ (Theorem 2.6), we see that $p_{t}(x, y) \geq A P^{x}\left(\tau_{y} \leq t\right)$, and hence, setting $g_{n}(z$, $x, y) \equiv\left(\left(\frac{2}{5}\right)^{n} z\right)^{\frac{1}{d w-1}} \log p_{\left(\frac{2}{5}\right)^{n} z}(x, y)$,

$$
-\limsup _{n \rightarrow \infty}\left(\left(\frac{2}{5}\right)^{n} z\right)^{\frac{1}{d w-1}} \log P^{x}\left(\tau_{y} \leq\left(\frac{2}{5}\right)^{n} z\right) \geq-\limsup _{n \rightarrow \infty} g_{n}(z, x, y) \text {. }
$$


Now, let $D_{n}(y) \equiv\{C: C$ is an $n$-complex which contains $y\}, D_{n}^{1}(y) \equiv D_{n}(y)$ $\cup\left\{C: C\right.$ is an $n$-complex which is connected to $\left.D_{n}(y)\right\}$. As $x \neq y, x \in D_{n}^{1}(y)$ for large $n$. Denote $b_{i}^{n}\left(1 \leq i \leq t_{0}\left(\exists t_{0}\right)\right)$ the boundary of $D_{n}^{1}(y)$. Then, by Theorem 2.6, $M_{n} \equiv \max _{i, 0 \leq s \leq t} p_{t-s}\left(b_{i}^{n}, y\right)<\infty$ for $t \leq 1$. Hence,

$$
\begin{aligned}
p_{t}(x, y) & \leq \sum_{i} P^{x}\left(\tau_{b_{i}^{n}}<\tau_{y}\right) \int_{0}^{t} p_{t-s}\left(b_{i}^{n}, y\right) P^{x}\left(\tau_{b_{i}^{n}} \in d s \mid \tau_{b_{i}^{n}}<\tau_{y}\right) \\
& \leq M_{n} \max _{i} P^{x}\left(\tau_{b_{i}^{n}} \leq t\right) .
\end{aligned}
$$

Thus,

$$
-\min _{i} \liminf _{n \rightarrow \infty}\left(\left(\frac{2}{5}\right)^{n} z\right)^{\frac{1}{d w-1}} \log P^{x}\left(\tau_{b_{n}^{n}} \leq\left(\frac{2}{5}\right)^{n} z\right) \leq-\liminf _{n \rightarrow \infty} g_{n}(z, x, y) .
$$

Taking $n \rightarrow \infty$, we have (18) with $y$ instead of $b_{i}^{n}$, as $P^{x}\left(\tau_{b_{i}^{n}} \leq t\right)$ converges compact uniformly to $P^{x}\left(\tau_{y} \leq t\right)$ for small $t$. We now obtain the result.

Corollary 4.1. There is no function $f: E \times E \rightarrow \mathbf{R}_{+}$which satisfies the following for some bounded function $G$ :

$$
-\lim _{t \rightarrow 0} G(t) t^{\frac{1}{d_{w}-1}} \log p_{t}(x, y)=f(x, y) \quad \forall x, y \in E .
$$

Proof. Let us assume that (19) holds for some $f$ and deduce contradiction. For $x, y$ with $d(x, y)=1$, we have from (1)

$$
-\lim _{n \rightarrow \infty} L(z)\left(\left(\frac{2}{5}\right)^{n} z\right)^{\frac{1}{d w-1}} \log p_{\left(\frac{2}{5}\right)^{n} z}(x, y)=1 \quad \forall z>0,
$$

where $L(z) \equiv F(z)^{-1}$. Thus, if (19) holds, then $\lim _{n \rightarrow \infty} G\left(\left(\frac{2}{5}\right)^{n} z\right)=f(x, y) L(z)$ so that

$$
\exists \widetilde{G}(z)=\lim _{n \rightarrow \infty} G\left(\left(\frac{2}{5}\right)^{n} z\right)
$$

Then, $\widetilde{G}(z) / L(z)=f(x, y)$. As the left hand side of the equation does not depend on $x, y$ we can take

(21) $\widetilde{G}(z)=L(z)$ and $f(x, y)=1$ for all $x, y \in E$ with $d(x, y)=1$.

Using (20) and (21), we can write (19) for general $x, y \in E$ as

$$
-\lim _{n \rightarrow \infty} L(z)\left(\left(\frac{2}{5}\right)^{n} z\right)^{\frac{1}{d w-1}} \log p_{\left(\frac{2}{5}\right)^{n} z}(x, y)=f(x, y) .
$$

On the other hand, according to (1), the right hand side of (22) is 
$d(x, y)^{\frac{d w}{d w-1}} \cdot F\left(\frac{z}{d(x, y)}\right) / F(z)$, which depends on $z$ by a suitable choice of $d(x, y)$ as $F$ is not a constant. This is a contradiction.

We next treat the non-lattice case. In fact, Theorem $1.2 \mathrm{~b}$ ) holds for the class of affine nested fractals we treat with the condition that $\sum_{k=1}^{k_{0}} \mathbb{Z} \log \tau_{k}$ is a dense subgroup of $\mathbf{R}$. We will prove it in the following.

Proof of Theorem 1.2 b). By Lemma 3.3, $\lim _{s \rightarrow \infty} L(s)$ exists. From Lemma 3.4, using Brujin's exponential type Tauberian theorem (see, for example [6]), we have

$$
-\lim _{t \rightarrow 0} t^{\frac{1}{d w-1}} \log P^{x}\left(\tau_{y} \leq t\right)=c_{4.1} d(x, y)^{\frac{d w}{d w-1}}
$$

where $c_{4.1}$ is some positive constant. By a suitable normalization of the metric $d$, we can take $c_{4.1}=1$. We then obtain 1) by the same argument as in the last part of the proof of Theorem $1.2 \mathrm{a}$ ). On the other hand, for $0 \leq \alpha \leq \beta \leq T$, we have by Hölder's inequality

$$
d(x, y) \leq \int_{\alpha}^{\beta}\left|\frac{d}{d t} d(x, \phi(t))\right| d t \leq\left(\int_{\alpha}^{\beta}\left|\frac{d}{d t} d(x, \phi(t))\right|^{\frac{d w}{d w-1}} d t\right)^{\frac{d w-1}{d w}}(\beta-\alpha)^{\frac{1}{d w}}
$$

With a suitable parametrization, we can construct $\phi:[0, T] \rightarrow E$ for which the equality holds. Thus

$$
\inf _{\phi: \phi(\alpha)=x, \phi(\beta)=y} I_{x}(\phi)=\left(\frac{d(x, y)}{(\beta-\alpha)^{\frac{1}{d w}}}\right)^{\frac{d w}{d w-1}} .
$$

Using 1) and (23), 2) can be obtained by a standard argument due to Varadhan ([20]).

We note that Theorem 1.2 holds for the heat kernel $\bar{p}_{t}(x, y)$ on the unbounded fractal introduced in the remark in the end of Section 2. The proof for the unbounded case is just easy modifications of the proof we did in this paper. Finally, we show the following.

Theorem 4.2. In the Theorem $1.1, L(t)$ can be chosen as a constant.

Proof. According to Fujita ([8]), $L(t)$ in Theorem 1.1 is obtained from another slowly varying function $L^{*}$ via a Tauberian theorem. This $L^{*}$ is the same function as $L(s)$ in Lemma 3.3 if we set $d_{w}=\frac{1+s}{s}, \tau_{1}=\frac{2}{1-p}, \tau_{2}=\frac{2}{p}$. The apriori estimates (8) is obtained in (4.12) of [8] and (7) holds in this case as $E$ is an interval. Thus, by the same argument as in the proof of Lemma 3.3, we see 
$\lim _{t \rightarrow \infty} L^{*}(t)>0$ exists so that $L(t)$ can be chosen as a constant.

\section{References}

[1] Barlow, M. T., Random walks, electrical resistance, and nested fractals, In : K. D. Elworthy, N. Ikeda (eds.) Asymptotic Problems in Probability Theory: stochastic models and diffusions on fractals, Montreal : Pitman (1993), 131-157.

[2] Barlow, M. T. and Bass, R. F., Transition densities for Brownian motion on the Sierpinski carpet, Probab. Theory Relat. Fields, 91 (1991), 307-330.

[3] Barlow, M. T. and Perkins, E. A., Brownian motion on the Sierpinski gasket, Probab. Theory Relat. Fields, 79 (1988), 543-624.

[4] Biggins, J. D. and Bingham, N. H., Near-constancy phenomena in branching processes, Math. Proc. Camb. Phil. Soc., 110 (1991), 545-558.

[5] L Large deviations in the supercritical branching process, Adv. Appl. Prob., 25 (1993), 757-772.

[6] Bingham, N. H., Goldie, C. M. and Teugels, J. L., Regular variation, Encyclopedia of mathematics and its applications, Cambridge Univ. Press., Cambridge, 1987.

[7] Fitzsimmons, P. J., Hambly, B. M. and Kumagai, T., Transition density estimates for Brownian motion on affine nested fractals, Comm. Math. Phys., 165 (1994), 595-620.

[8] Fujita, T., Some asymptotic estimates of transition probability densities for generalized diffusion processes with self-similar speed measures, Publ. RIMS, Kyoto Univ., 26 (1990), 819-840.

[9] Fujita, T. and Watanabe, S., Large deviations for one-dimensional diffusion processes with self-similar speed measures, Preprint.

[10] Fukushima, M., Dirichlet forms, diffusion processes and spectral dimensions for nested fractals, In: S. Albeverio, et al. (eds.) Ideas and Methods in Mathematical Analysis, Stochastics, and Applications, In Memory of R. H $\phi$ egh-Krohn, vol. 1, Cambridge Univ. Press (1992), 151-161.

[11] Hutchinson, John E., Fractals and self similarity, Indiana Univ. Math. J., 30 (1981), 713747.

[12] Kigami, J., Harmonic calculus on P.C.F. self-similar sets, Trans. Amer. Math. Soc., 335 (1993), 721-755.

[13] Kigami, J. and Lapidus, M. L., Weyl's spectral problem for the spectral distribution of Laplacians on P.C.F. self-similar fractals, Comm. Math. Phys., 158 (1993), 93-125.

[14] Kotani, S. and Watanabe, S., Krein's spectral theory of strings and generalized diffusion processes, In : M. Fukushima (ed.) Functional analysis in Markov processes, Lect. Notes Math., 923, Springer (1982), 235-259.

[15] Kumagai, T., Estimates of the transition densities for Brownian motion on nested fractals, Probab. Theory Relat. Fields, 96 (1993), 205-224.

[16] Kusuoka, S., Diffusion processes on nested fractals, In : Dobrushin, R. L., Kusuoka, S. : Statistical Mechanics and Fractals, Lect. Notes Math., 1567, Springer (1993).

[17] Lindstr $\phi$ m, T., Brownian motion on nested fractals, Memoirs Amer. Math. Soc. No. 420, 83, (1990).

[18] Schilder, M., On a Laplace asymptotic formula for Wiener integrals, Trans. Amer. Math. Soc., 125 (1966), 63-85.

[19] Varadhan, S., On the behavior of the fundamental solution of the heat equation with variable coefficients, Comm. Pure Appl. Math., 20 (1967), 431-455.

[20] _ Diffusion processes in a small time interval, Comm. Pure Appl. Math., 20 (1967), 659 
-685 .

[21] Watanabe, S., One dimensional diffusion processes and large deviation (in Japanese), Suuri kagaku, 380 (1995), 9-14.

Note added in proof : After the manuscript was submitted, the author was informed from Professor Watanabe that there was a preprint on large deviations for onedimensional diffusion processes with self-similar speed measures ([9]). 